\title{
Degree powers in graphs with forbidden subgraphs
}

\author{
Béla Bollobás*ił and Vladimir Nikiforov* \\ Submitted: Jan 27, 2004; Accepted: Jun 10, 2004; Published: Jun 25, 2004 \\ MR Subject Classifications: 05C35
}

Abstract
For every real $p>0$ and simple graph $G$, set
\[ f(p, G)=\sum_{u \in V(G)} d^{p}(u), \]

and let $\phi(r, p, n)$ be the maximum of $f(p, G)$ taken over all $K_{r+1}$-free graphs $G$ of order $n$. We prove that, if $0<p<r$, then

$$
\phi(r, p, n)=f\left(p, T_{r}(n)\right),
$$

where $T_{r}(n)$ is the $r$-partite Turan graph of order $n$. For every $p \geq r+\lceil\sqrt{2 r}\rceil$ and $n$ large, we show that

$$
\phi(p, n, r)>(1+\varepsilon) f\left(p, T_{r}(n)\right)
$$

for some $\varepsilon=\varepsilon(r)>0$.

Our results settle two conjectures of Caro and Yuster.

\section{Introduction}

Our notation and terminology are standard (see, e.g. [1]).

Caro and Yuster [3] introduced and investigated the function

$$
f(p, G)=\sum_{u \in V(G)} d^{p}(u),
$$

where $p \geq 1$ is integer and $G$ is a graph. Writing $\phi(r, p, n)$ for the maximum value of $f(p, G)$ taken over all $K_{r+1}$-free graphs $G$ of order $n$, Caro and Yuster stated that, for every $p \geq 1$,

$$
\phi(r, p, n)=f\left(p, T_{r}(n)\right)
$$

\footnotetext{
${ }^{*}$ Department of Mathematical Sciences, University of Memphis, Memphis TN 38152, USA

${ }^{\dagger}$ Trinity College, Cambridge CB2 1TQ, UK

${ }^{\ddagger}$ Research supported in part by DARPA grant F33615-01-C-1900.
} 
where $T_{r}(n)$ is the $r$-partite Turán graph of order $n$. Although true for $p=2, r \geq 2$, simple examples show that (1) fails for every fixed $r \geq 2$ and all sufficiently large $p$ and $n$; this was observed by Schelp [4]. A natural problem arises: given $r \geq 2$, determine those real values $p>0$, for which equality (1) holds. Furthermore, determine the asymptotic value of $\phi(r, p, n)$ for large $n$.

In this note we essentially answer these questions. In Section 2 we prove that (1) holds whenever $0<p<r$ and $n$ is large. Next, in Section 3, we describe the asymptotic structure of $K_{r+1}$-free graphs $G$ of order $n$ such that $f(p, G)=\phi(r, p, n)$. We deduce that, if $p \geq r+\lceil\sqrt{2 r}\rceil$ and $n$ is large, then

$$
\phi(r, p, n)>(1+\varepsilon) f\left(p, T_{r}(n)\right)
$$

for some $\varepsilon=\varepsilon(r)>0$. This disproves Conjecture 6.2 in [3]. In particular,

$$
\frac{r}{p e} \geq \frac{\phi(r, p, n)}{n^{p+1}} \geq \frac{r-1}{(p+1) e}
$$

holds for large $n$, and therefore, for any fixed $r \geq 2$,

$$
\lim _{n \rightarrow \infty} \frac{\phi(r, p, n)}{f\left(p, T_{r}(n)\right)}
$$

grows exponentially in $p$.

The case $r=2$ is considered in detail in Section 4; we show that, if $r=2$, equality (1) holds for $0<p \leq 3$, and is false for every $p>3$ and $n$ large.

In Section 5 we extend the above setup. For a fixed $(r+1)$-chromatic graph $H$, $(r \geq 2)$, let $\phi(H, p, n)$ be the maximum value of $f(p, G)$ taken over all $H$-free graphs $G$ of order $n$. It turns out that, for every $r$ and $p$,

$$
\phi(H, p, n)=\phi(r, p, n)+o\left(n^{p+1}\right) .
$$

This result completely settles, with the proper changes, Conjecture 6.1 of [3]. In fact, Pikhurko [5] proved this for $p \geq 1$, although he incorrectly assumed that (1) holds for all sufficiently large $n$.

\section{The function $\phi(r, p, n)$ for $p<r$}

In this section we shall prove the following theorem.

Theorem 1 For every $r \geq 2,0<p<r$, and sufficiently large $n$,

$$
\phi(r, p, n)=f\left(p, T_{r}(n)\right) .
$$

Proof Erdös [2] proved that, for every $K_{r+1}$-free graph $G$, there exists an $r$-partite graph $H$ with $V(H)=V(G)$ such that $d_{G}(u) \leq d_{H}(u)$ for every $u \in V(G)$. As Caro and Yuster 
noticed, this implies that, for $K_{r+1}$-free graphs $G$ of order $n$, if $f(p, G)$ attains a maximum then $G$ is a complete $r$-partite graph. Every complete $r$-partite graph is defined uniquely by the size of its vertex classes, that is, by a vector $\left(n_{i}\right)_{1}^{r}$ of positive integers satisfying $n_{1}+\ldots+n_{r}=n$; note that the Turán graph $T_{r}(n)$ is uniquely characterized by the condition $\left|n_{i}-n_{j}\right| \leq 1$ for every $i, j \in[r]$. Thus we have

$$
\phi(r, p, n)=\max \left\{\sum_{i=1}^{r} n_{i}\left(n-n_{i}\right)^{p}: n_{1}+\ldots+n_{r}=n, 1 \leq n_{1} \leq \ldots \leq n_{r}\right\} .
$$

Let $\left(n_{i}\right)_{1}^{r}$ be a vector on which the value of $\phi(r, p, n)$ is attained. Routine calculations show that the function $x(n-x)^{p}$ increases for $0 \leq x \leq \frac{n}{p+1}$, decreases for $\frac{n}{p+1} \leq x \leq n$, and is concave for $0 \leq x \leq \frac{2 n}{p+1}$. If $n_{r} \leq\left\lfloor\frac{2 n}{p+1}\right\rfloor$, the concavity of $x(n-x)^{p}$ implies that $n_{r}-n_{1} \leq 1$, and the proof is completed, so we shall assume $n_{r}>\left\lfloor\frac{2 n}{p+1}\right\rfloor$. Hence we deduce

$$
n_{1}(r-1)+\left\lfloor\frac{2 n}{p+1}\right\rfloor<n_{1}+\ldots+n_{r}=n .
$$

We shall also assume

$$
n_{1} \geq\left\lfloor\frac{n}{p+1}\right\rfloor,
$$

since otherwise, adding 1 to $n_{r}$ and subtracting 1 from $n_{1}$, the value $\sum_{i=1}^{r} n_{i}\left(n-n_{i}\right)^{p}$ will increase, contradicting the choice of $\left(n_{i}\right)_{1}^{r}$. Notice that, as $n_{1} \leq n / r$, inequality (5) is enough to prove the assertion for $p \leq r-1$ and every $n$. From (4) and (5), we obtain that

$$
(r-1)\left\lfloor\frac{n}{p+1}\right\rfloor+\left\lfloor\frac{2 n}{p+1}\right\rfloor<n .
$$

Letting $n \rightarrow \infty$, we see that $p \geq r$, contradicting the assumption and completing the proof.

Maximizing independently each summand in (3), we see that, for every $r \geq 2$ and $p>0$,

$$
\phi(r, p, n) \leq \frac{r}{p+1}\left(\frac{p}{p+1}\right)^{p} n^{p+1} .
$$

\section{The asymptotics of $\phi(r, p, n)$}

In this section we find the asymptotic structure of $K_{r+1}$-free graphs $G$ of order $n$ satisfying $f(p, G)=\phi(r, p, n)$, and deduce asymptotic bounds on $\phi(r, p, n)$.

Theorem 2 For all $r \geq 2$ and $p>0$, there exists $c=c(p, r)$ such that the following assertion holds.

If $f(p, G)=\phi(r, p, n)$ for some $K_{r+1}$-free graph $G$ of order $n$, then $G$ is a complete $r$-partite graph having $r-1$ vertex classes of size $\mathrm{cn}+o(n)$. 
Proof We already know that $G$ is a complete $r$-partite graph; let $n_{1} \leq \ldots \leq n_{r}$ be the sizes of its vertex classes and, for every $i \in[r]$, set $y_{i}=n_{i} / n$. It is easy to see that

$$
\phi(r, p, n)=\psi(r, p) n^{p+1}+o\left(n^{p+1}\right),
$$

where the function $\psi(r, p)$ is defined as

$$
\psi(r, p)=\max \left\{\sum_{i=1}^{r} x_{i}\left(1-x_{i}\right)^{p} \quad: x_{1}+\ldots+x_{r}=1,0 \leq x_{1} \leq \ldots \leq x_{r}\right\}
$$

We shall show that if the above maximum is attained at $\left(x_{i}\right)_{1}^{r}$, then $x_{1}=\ldots=$ $x_{r-1}$. Indeed, the function $x(1-x)^{p}$ is concave for $0 \leq x \leq 2 /(p+1)$, and convex for $2 /(p+1) \leq x \leq 1$. Hence, there is at most one $x_{i}$ in the interval $(2 /(p+1) \leq x \leq 1]$, which can only be $x_{r}$. Thus $x_{1}, \ldots, x_{r-1}$ are all in the interval $[0,2 /(p+1)]$, and so, by the concavity of $x(1-x)^{p}$, they are equal. We conclude that, if

$$
0 \leq x_{1} \leq \ldots \leq x_{r}, x_{1}+\ldots+x_{r}=1
$$

and $x_{j}>x_{i}$ for some $1 \leq i<j \leq r-1$, then $\sum_{i=1}^{r} x_{i}\left(1-x_{i}\right)^{p}$ is below its maximum value. Applying this conclusion to the numbers $\left(y_{i}\right)_{1}^{r}$, we deduce the assertion of the theorem.

Set

$$
g(r, p, x)=(r-1) x(1-x)^{p}+(1-(r-1) x)(r x)^{p} .
$$

From the previous theorem it follows that

$$
\psi(r, p)=\max _{0 \leq x \leq 1 /(r-1)} g(r, p, x) .
$$

Finding $\psi(r, p)$ is not easy when $p>r$. In fact, for some $p>r$, there exist $0<x<y<1$ such that

$$
\psi(r, p)=g(r, p, x)=g(r, p, y) .
$$

In view of the original claim concerning (1), it is somewhat surprising, that for $p>$ $2 r-1$, the point $x=1 / r$, corresponding to the Turán graph, not only fails to be a maximum of $g(r, p, x)$, but, in fact, is a local minimum.

Observe that

$$
\frac{f\left(p, T_{r}(n)\right)}{n^{p+1}}=\left(\frac{r-1}{r}\right)^{p}+o(1),
$$

so, to find for which $p$ the function $\phi(r, p, n)$ is significantly greater than $f\left(p, T_{r}(n)\right)$, we shall compare $\psi(r, p)$ to $\left(\frac{r-1}{r}\right)^{p}$.

Theorem 3 Let $r \geq 2, p \geq r+\lceil\sqrt{2 r}\rceil$. Then

$$
\psi(r, p)>(1+\varepsilon)\left(\frac{r-1}{r}\right)^{p}
$$

for some $\varepsilon=\varepsilon(r)>0$. 
Proof We have

$$
\begin{aligned}
\psi(r, p) & \geq g\left(r, p, \frac{1}{p}\right)=\frac{r-1}{p}\left(\frac{p-1}{p}\right)^{p}+\left(1-\frac{r-1}{p}\right)\left(\frac{r-1}{p}\right)^{p} \\
& >\frac{r-1}{p}\left(\frac{p-1}{p}\right)^{p} .
\end{aligned}
$$

To prove the theorem, it suffices to show that

$$
\frac{r-1}{p}\left(\frac{(p-1) r}{p(r-1)}\right)^{p}>1+\varepsilon
$$

for some $\varepsilon=\varepsilon(r)>0$. Routine calculations show that

$$
\frac{r-1}{p}\left(1+\frac{p-r}{p(r-1)}\right)^{p}
$$

increases with $p$. Thus, setting $q=\lceil\sqrt{2 r}\rceil$, we find that

$$
\begin{aligned}
& \frac{r-1}{p}\left(1+\frac{p-r}{p(r-1)}\right)^{p} \\
& \geq \frac{r-1}{r+q}\left(1+\left(\begin{array}{c}
r+q \\
1
\end{array}\right) \frac{q}{(r+q)(r-1)}+\left(\begin{array}{c}
r+q \\
2
\end{array}\right) \frac{q^{2}}{(r+q)^{2}(r-1)^{2}}\right) \\
& =\frac{r-1}{r+q}+\frac{q}{r+q}+\frac{q^{2}(r+q-1)}{2(r+q)^{2}(r-1)} \geq 1-\frac{1}{r+q}+\frac{r(r+q-1)}{(r+q)^{2}(r-1)} \\
& =1+\frac{r(r+q-1)-(r+q)(r-1)}{(r+q)^{2}(r-1)}=1+\frac{q}{(r+q)^{2}(r-1)} .
\end{aligned}
$$

Hence, (7) holds with

$$
\varepsilon=\frac{\lceil\sqrt{2 r}\rceil}{(r+\lceil\sqrt{2 r}\rceil)^{2}(r-1)}
$$

completing the proof.

We have, for $n$ sufficiently large,

$$
\begin{aligned}
\frac{\phi(r, p, n)}{n^{p+1}} & =\psi(r, p)+o(1) \geq g\left(r, p, \frac{1}{p+1}\right)+o(1) \\
& =\frac{r-1}{p+1}\left(\frac{p}{p+1}\right)^{p}+\left(1-\frac{r-1}{p+1}\right)\left(\frac{r-1}{p+1}\right)^{p}+o(1) \\
& >\frac{r-1}{p+1}\left(\frac{p}{p+1}\right)^{p} .
\end{aligned}
$$

Hence, in view of (6), we find that, for $n$ large,

$$
\frac{r}{p e} \geq \frac{r}{p}\left(\frac{p}{p+1}\right)^{p+1} \geq \frac{\phi(r, p, n)}{n^{p+1}} \geq \frac{r-1}{p+1}\left(\frac{p}{p+1}\right)^{p} \geq \frac{(r-1)}{(p+1) e} .
$$


In particular, we deduce that, for any fixed $r \geq 2$,

$$
\lim _{n \rightarrow \infty} \frac{\phi(r, p, n)}{f\left(p, T_{r}(n)\right)}
$$

grows exponentially in $p$.

\section{Triangle-free graphs}

For triangle-free graphs, i.e., $r=2$, we are able to pinpoint the value of $p$ for which (1) fails, as stated in the following theorem.

Theorem 4 If $0<p \leq 3$ then

$$
\phi(3, p, n)=f\left(p, T_{2}(n)\right) .
$$

For every $\varepsilon>0$, there exists $\delta$ such that if $p>3+\delta$ then

$$
\phi(3, p, n)>(1+\varepsilon) f\left(p, T_{2}(n)\right)
$$

for $n$ sufficiently large.

Proof We start by proving (8). From the proof of Theorem 1 we know that

$$
\phi(p, n, 3)=\max _{k \in\lceil n / 2\rceil}\left\{k(n-k)^{p}+(n-k) k^{p}\right\} .
$$

Our goal is to prove that the above maximum is attained at $k=\lceil n / 2\rceil$.

If $0<p \leq 2$, the function $x(1-x)^{p}$ is concave, and (8) follows immediately.

Next, assume that $2<p \leq 3$; we claim that the function

$$
g(x)=(1+x)(1-x)^{p}+(1-x)(1+x)^{p}
$$

is concave for $|x| \leq 1$. Indeed, we have

$$
\begin{aligned}
g(x) & =\left(1-x^{2}\right)\left((1-x)^{p-1}+(1+x)^{p-1}\right)=2\left(1-x^{2}\right) \sum_{i=0}^{\infty}\left(\begin{array}{c}
p-1 \\
2 i
\end{array}\right) x^{2 i} \\
& =2+2 \sum_{i=1}^{\infty}\left(\left(\begin{array}{c}
p-1 \\
2 i
\end{array}\right)-\left(\begin{array}{c}
p-1 \\
2 i-2
\end{array}\right)\right) x^{2 i} \\
& =2+2 \sum_{i=1}^{\infty}\left(\begin{array}{c}
p-1 \\
2 i-2
\end{array}\right)\left(\frac{(p-2 i-1)(p-2 i-2)}{(2 i-1) 2 i}-1\right) x^{2 i}
\end{aligned}
$$

Since, for every $i$, the coefficient of $x^{2 i}$ is nonpositive, the function $g(x)$ is concave, as claimed. 
Therefore, the function $h(x)=x(n-x)^{p}+(n-x) x^{p}$ is concave for $1 \leq x \leq n$. Hence, for every integer $k \in[n]$, we have

$$
\begin{aligned}
h\left(\left\lceil\frac{n}{2}\right\rceil\right)+h\left(\left\lfloor\frac{n}{2}\right\rfloor\right) & \geq h(k)+h(n-k)=2 h(k) \\
& =2\left(k(n-k)^{p}+(n-k) k^{p}\right),
\end{aligned}
$$

proving (8).

Inequality (9) follows easily, since, in fact, for every $p>3$, the function $g(x)$ has a local minimum at 0 .

\section{$5 \quad H$-free graphs}

In this section we are going to prove the following theorem.

Theorem 5 For every $r \geq 2$, and $p>0$,

$$
\phi(H, p, n)=\phi(r, p, n)+o\left(n^{p+1}\right) .
$$

A few words about this theorem seem in place. As already noted, Pikhurko [5] proved the assertion for $p \geq 1$; although he incorrectly assumed that (1) holds for all $p$ and sufficiently large $n$, his proof is valid, since it is independent of the exact value of $\phi(r, p, n)$. Our proof is close to Pikhurko's, and is given only for the sake of completeness.

We shall need the following theorem (for a proof see, e.g., [1], Theorem 33, p. 132).

Theorem 6 Suppose $H$ is an $(r+1)$-chromatic graph. Every $H$-free graph $G$ of sufficiently large order $n$ can be made $K_{r+1}$-free by removing o $\left(n^{2}\right)$ edges.

Proof of Theorem 5 Select a $K_{r+1}$-free graph $G$ of order $n$ such that $f(p, G)=$ $\phi(r, p, n)$. Since $G$ is $r$-partite, it is $H$-free, so we have $\phi(H, p, n) \geq \phi(r, p, n)$. Let now $G$ be an $H$-free graph of order $n$ such that

$$
f(p, G)=\phi(H, p, n) .
$$

Theorem 6 implies that there exists a $K_{r+1}$-free graph $F$ that may be obtained from $G$ by removing at most $o\left(n^{2}\right)$ edges. Obviously, we have

$$
e(G)=e(F)+o\left(n^{2}\right) \leq \frac{r-1}{2 r} n^{2}+o\left(n^{2}\right) .
$$

For $0<p \leq 1$, by Jensen's inequality, we have

$$
\left(\frac{1}{n} f(p, G)\right)^{1 / p} \leq \frac{1}{n} f(1, G)=\frac{1}{n} 2 e(G) \leq \frac{r-1}{r} n+o(n) .
$$


Hence, we find that

$$
f(p, G) \leq\left(\frac{r-1}{r}\right)^{p} n^{p+1}+o\left(n^{p+1}\right)=\phi(r, p, n)+o\left(n^{p+1}\right),
$$

completing the proof.

Next, assume that $p>1$. Since the function $x n^{p-1}-x^{p}$ is decreasing for $0 \leq x \leq n$, we find that

$$
d_{G}^{p}(u)-d_{F}^{p}(u) \leq\left(d_{G}(u)-d_{F}(u)\right) n^{p-1}
$$

for every $u \in V(G)$. Summing this inequality for all $u \in V(G)$, we obtain

$$
\begin{aligned}
f(p, G) & \leq f(p, F)+\left(d_{G}(u)-d_{F}(u)\right) n^{p-1}=f(p, F)+o\left(n^{p+1}\right) \\
& \leq \phi(r, p, n)+o\left(n^{p+1}\right),
\end{aligned}
$$

completing the proof.

\section{Concluding remarks}

It seems interesting to find, for each $r \geq 3$, the minimum $p$ for which the equality (1) is essentially false for $n$ large. Computer calculations show that this value is roughly 4.9 for $r=3$, and 6.2 for $r=4$, suggesting that the answer might not be easy.

\section{References}

[1] B. Bollobás, Modern Graph Theory, Graduate Texts in Mathematics, 184, SpringerVerlag, New York (1998), xiv+394 pp.

[2] P. Erdős, On the graph theorem of Turán (in Hungarian), Mat. Lapok 21 (1970), 249-251.

[3] Y. Caro and R. Yuster, A Turán type problem concerning the powers of the degrees of a graph, Electron. J. Comb. 7 (2000), RP 47.

[4] R. H. Schelp, review in Math. Reviews, MR1785143 (2001f:05085), 2001.

[5] O. Pikhurko, Remarks on a Paper of Y. Caro and R. Yuster on Turán problem, preprint, arXiv:math.CO/0101235v129 Jan 2001. 